\title{
A Polymer Film Dye Laser with Spatially Modulated Emission Controlled by Transversely Distributed Pumping
}

\author{
Zurab V. Wardosanidze, Andro Chanishvili, and Guram Chilaya \\ V. Chavchanidze Institute of Cybernetics of the Georgian Technical University, 5 Sandro Euli St., 0186 Tbilisi, Georgia \\ Correspondence should be addressed to Zurab V. Wardosanidze; zvward@yahoo.com
}

Received 16 June 2016; Revised 24 August 2016; Accepted 5 September 2016

Academic Editor: Giancarlo C. Righini

Copyright ( 2016 Zurab V. Wardosanidze et al. This is an open access article distributed under the Creative Commons Attribution License, which permits unrestricted use, distribution, and reproduction in any medium, provided the original work is properly cited.

\begin{abstract}
Spatial modulation of laser emission controlled by the structure of excitation light field was demonstrated. A dye doped polymer film as an active medium was sandwiched between two laser mirrors forming a laser cell. The pumping was performed by an interference pattern formed with two mutually coherent beams of the second harmonic of a Q-switched Nd:YAG laser (532 nm) and located in the plane of the laser cell. The laser emission was observed normally on the plane of the cell. The cross section of the obtained laser emission was modulated in intensity with an interval between maximums depending on the period of the pumping interference pattern. Thus, the emitted light field qualitatively looks like diffraction from an elementary dynamic hologram, that is, a holographic diffraction grating.
\end{abstract}

\section{Introduction}

An elementary hologram, for example, a holographic diffractive grating, usually represents a passive diffractive device. For obtaining the information about the recorded holographic structure an external light source is required [1]. However, as it is known, diffraction is the result of interference of secondary waves from all optical gaps of the periodical structure of the grating $[2,3]$. Therefore, if we have a structure whose areas emit mutually coherent light waves, the total light field will be analogous to a passive diffraction picture. Such a result was already observed during the study of cholesteric liquid crystal laser emission coherency [4]. In this case the excitation was performed in the form of an interference pattern of pumping beams.

The laser beams interference is used in various spheres of science and technology and, amongst all, for achieving laser emission. In particular, double beam coherent pumping has long been used to obtain a distributed feedback (DFB) in dye lasers [5-10]. In these cases the mutually coherent pumping beams in the active medium form an interference pattern whose bright and dark strips are distributed along the generated laser emission (Figure 1).
But the correlation between the emitting centers of separated emitting areas of the active medium allows one to use the double beam coherent pumping not only for the DFB formation. For instance, as it was shown recently for a CLC laser [4], the excitation by an interference pattern gives rise to the spatially modulated laser emission.

In the present paper, lasing from a dye doped polymer film at transversally distributed pumping (Figure 2) [11] is investigated. Analogous to [4], the mutually coherent pumping beams form in the active medium an interference pattern whose bright and dark strips are distributed perpendicular to the generated laser emission. The luminescent areas of the active medium being inside the cavity can generate laser emission separately. Due to correlation between the emitting centers of different lasing areas the conditions for interference of the beams from these areas arise. Therefore, the emission of such a laser should be spatially modulated and should form a pattern similar to diffraction from a dynamic holographic grating [1]. The aim of this study was to obtain and investigate the spatially modulated laser emission from a dye doped polymer film and to get an improved pattern of lasing by improving the laser emission coherency as compared with [4]. 


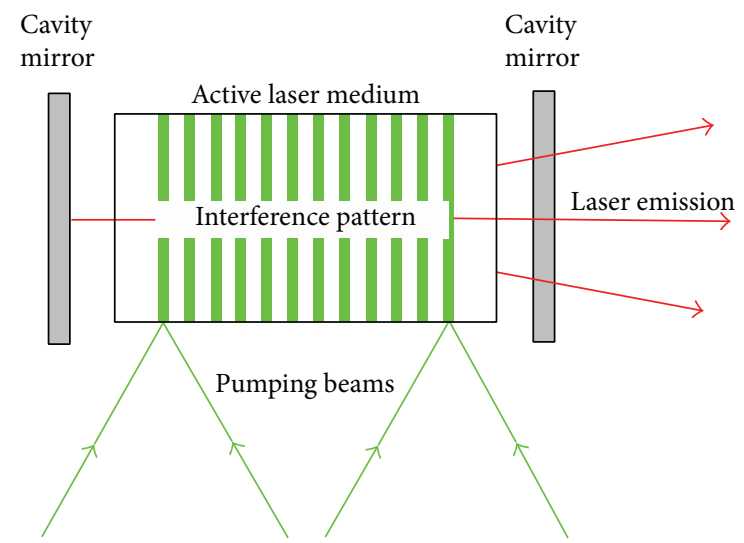

FIGURE 1: Dye laser with longitudinally distributed feedback (excitation).

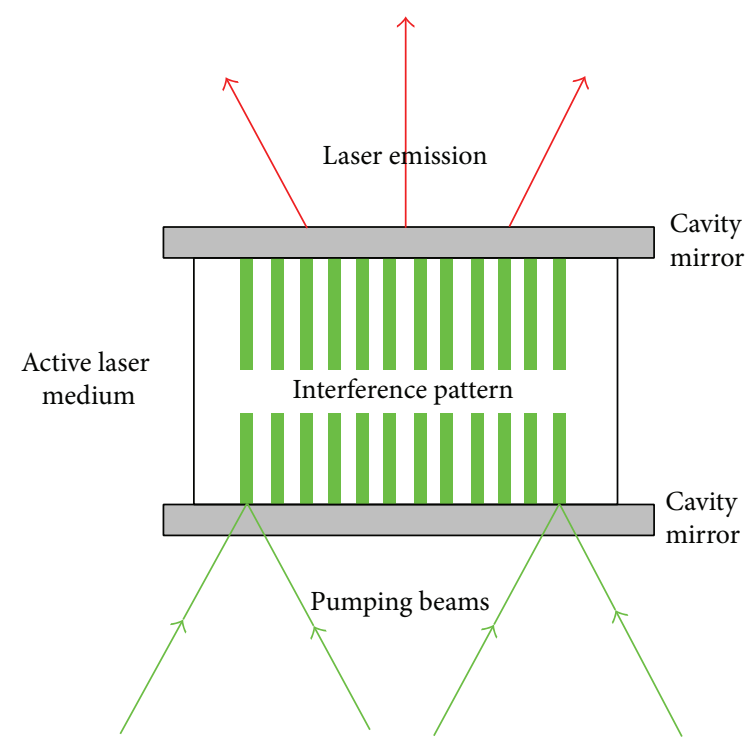

FIGURE 2: Dye laser with transversally distributed excitation.

\section{Experiment}

The experimental setup was the same as that used for holographic recording and for pumping of the DFB lasers mentioned above (Figure 3) [1, 4-10]. The second harmonic $(532 \mathrm{~nm})$ of a Q-switched Nd:YAG laser 1 with pulse duration of $15 \mathrm{~ns}$ was used for the coherent pumping. The repetition frequency of pulses was $12.5 \mathrm{~Hz}$. The laser provided the coherence length of approximately $100 \mathrm{~mm}$. With a beam splitter, the beam was divided into two beams of equal intensity. The beam splitter was composed of two interference mirrors 2 and 3 reflecting 50\% and 100\% accordingly. The distance between the mirrors $(15-20 \mathrm{~mm})$ ensured a stable interference pattern. The laser cell consisted of a polyvinyl alcohol (PVA) film 6 doped with Rhodamine-6G and sandwiched between cavity mirrors 4-5 with enough transparency $(\approx 75 \%)$ for the pumping emission. The total energy of the pumping radiation was $20-30 \mathrm{~mJ}$, so the real energy of the

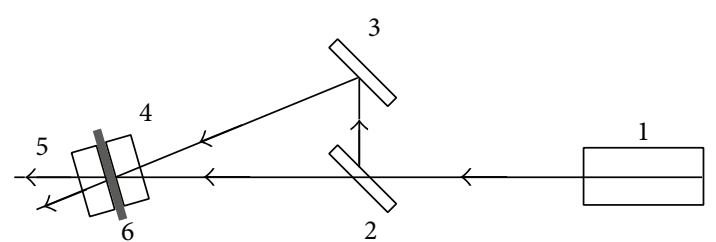

FIGURE 3: Scheme of experimental setup. 1, Nd:YAG laser; 2, semitransparent mirror; 3 , fully reflecting mirror; 4 and 5 , cavity mirrors of the laser cell; 6 , dye doped polymer film.

pulse (i.e., the energy incident on the laser cell) was 14$20 \mathrm{~mJ}$. The mirrors were placed with their reflective surfaces inside the cell contacting with the polymer layer. The radius of curvature of the concave mirror was $2 \mathrm{~m}$. The cell was oriented normally to the bisector of the pumping beams convergence angle. The pumping was carried out at beams convergence angles equal to $0.6^{\circ}, 0.9^{\circ}$, and $1.8^{\circ}$. Concentration of the dye was $0.148 \%$ and the thickness of the polymer film was $130 \mu \mathrm{m}$.

The pattern of the laser emission generated by the cell is shown in Figure 4. Figures 4(a), 4(b), and 4(c) correspond to the convergence angles of $0.6^{\circ}, 0.9^{\circ}$, and $1.8^{\circ}$, respectively. As can be seen, along the cross section of the light bundle there is no smooth distribution of intensity typical for conventional lasers. But the intensity has a spatially distributed form and qualitatively looks like a diffraction pattern from a diffraction grating. The angles between the intensity maximums' directions correspond to the following formula:

$$
\theta=2 \arcsin \frac{\lambda}{2 d}
$$

where, in our case, $\lambda$ is the wavelength of lasing and $d$ is the period of the pumping interference pattern. The diameter of the excited region was $1.5-2.0 \mathrm{~mm}$. In this area a sufficient number of the pumping interference pattern lines, that is, microlasers, were located. When shutting one of the pumping beams the modulation of laser emission pattern disappears (Figure 5). The elongated shape of the emitted light field in all photos results from the planoconcave cavity structure. To avoid Fabry-Perot interference of the generated emission, the pumping was performed not at the central but at the peripheral part of the resonator.

Due to nonlinear effects caused by the pumping, a dynamic phase or amplitude grating with modulation depth enough for observation of diffraction could be formed in the polymer film. To check this possibility, the lasing area was tested with a beam of He-Ne laser $(632 \mathrm{~nm})$. No signs of diffraction and thus no signs of any grating were detected.

The emitting spot structure was investigated under a microscope. In Figure 6 the photos of the spot demonstrating the modulated intensity of laser emission are shown. The convergence angles of the pumping beams were $0.6^{\circ}, 0.9^{\circ}$, and $1.8^{\circ}$, and spatial frequency of emitting areas was 19,28 , and 57 lines per millimeter accordingly. As seen, the laser emission is observed from all the pumped areas where the emission peaks are allocated as lasing stripes. Naturally, the peaks 


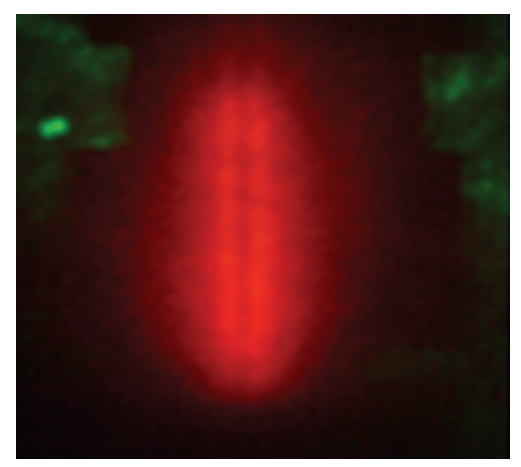

(a)

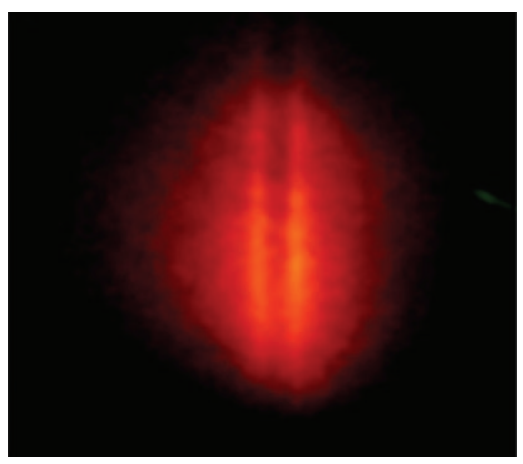

(b)

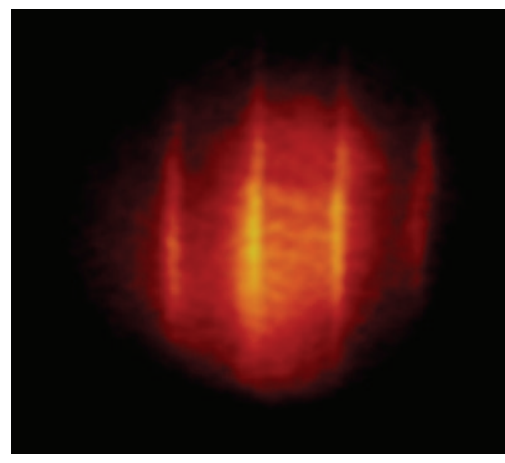

(c)

FIGURE 4: The emission pattern of the laser cell with double beam coherent transversally distributed pumping. The convergence angles are (a-c) $0.6^{\circ}, 0.9^{\circ}$, and $1.8^{\circ}$

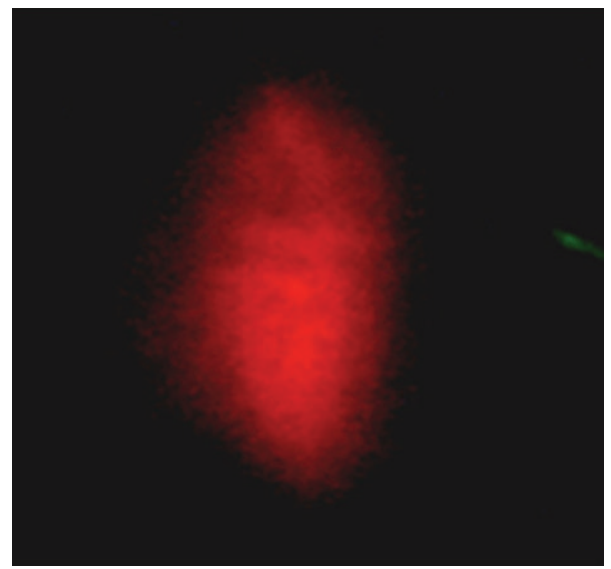

FIGURE 5: The emission pattern of the laser cell with single beam pumping.

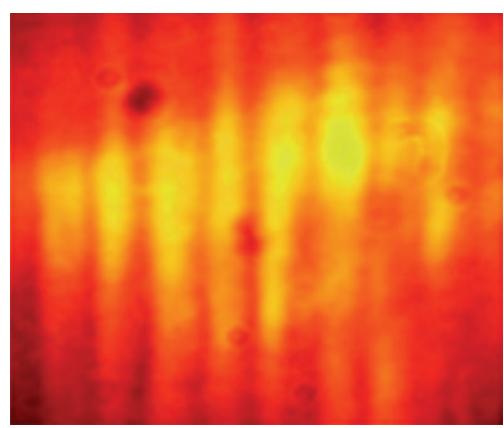

(a)

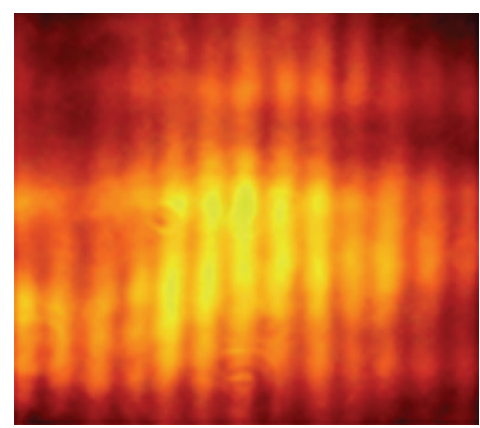

(b)

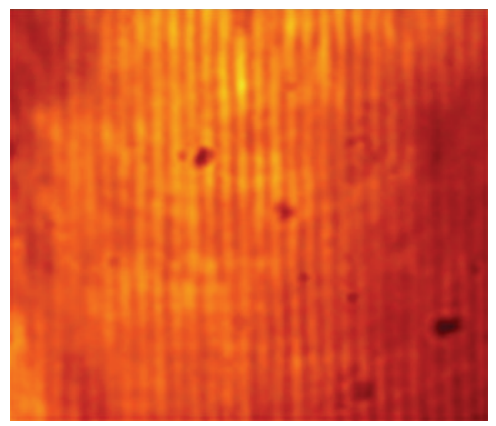

(c)

Figure 6: Microphotographs of the laser cell emitting area structure. The pumping beams convergence angles are $(\mathrm{a}-\mathrm{c}) 0.6^{\circ}, 0.9^{\circ}$, and $1.8^{\circ}$.

of lasing of these strips correspond to the intensity maximums of the interference pattern formed by the pumping beams.

In Figure 7 the laser emission spectrum is shown. The spectrum along the cross section of the beam of the radiation is strongly constant. The obtained spectrum of lasing is caused by the dye concentration, polymer matrix properties, and spectral reflection characteristics of the cavity mirrors.

\section{Discussion}

The aim of this study was to realize a laser with transversally distributed pumping performed by double beam coherent excitation of the dye doped polymer film (Figure 2). According to the authors' assumptions, the emission field of such a laser should be spatially modulated and must carry information about the spatial distribution of the excitation 


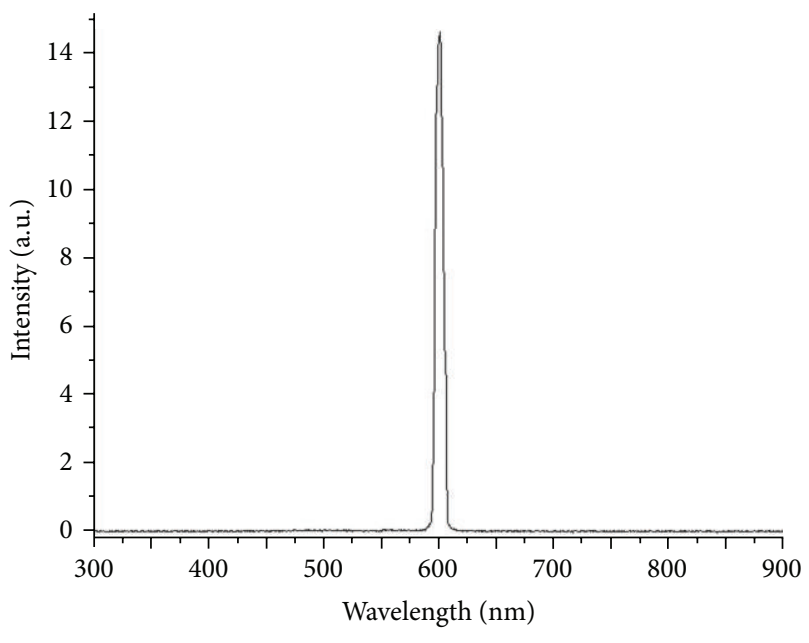

FIGURE 7: Spectrum of the laser emission.

field analogically to [4]. The results shown in Figure 4 confirmed these assumptions. In our opinion, the emitting spot represented a one-dimensional array of mutually coherent microlasers which gives the interference field.

As it can be seen from Figure 4, the emission patterns do not contain the central maximum of intensity. There have been observed only the intensity maximums located symmetrically with respect to the pattern center. So the cross section of the laser emission is not quite similar to the Bragg diffraction [1-3]. As it was noted above the absence of such a diffraction grating was confirmed by the absence of any signs of diffraction when probing the lasing area with a beam of He-Ne laser $(632 \mathrm{~nm})$. The interference of coherent microsheaves was able to form only symmetrically located intensity maximums. Therefore, we can say that the obtained pattern of emission is not a result of diffraction from a nonlinear grating formed in the active medium. The observed spatial modulation of lasing could only be the result of the interference of the mutually coherent microlasers emission. Thus, during the collective lasing of all strips, according to the Huygens-Fresnel principle $[2,3]$, the interference pattern shown in Figure 4 was formed. The obtained laser emission carries information about the periodical distribution of the pumping. Qualitatively it is almost similar to an elementary hologram whose diffraction orders also carry information about its periodical structure. So, we can say that the obtained laser operates like an active elementary dynamic hologram.

\section{Conclusion}

Thus, a dye doped polymer film laser with transversally distributed excitation is investigated. Similarly to [4] the emission pattern of this laser is spatially modulated. However, the intensity maximums in this case are more visible due to the enhanced lasing conditions. The intensity distribution of laser emission contains information about the pumping interference field as it takes place in the case of elementary dynamic hologram. But unlike the passive diffraction of incident light, the pattern is formed due to the own emission of the emitting areas.

Further we are planning to study the possibility of reconstruction of the image of a two-dimensional transparent object on the basis of such approach.

\section{Additional Points}

The following are the OCIS codes: (090.2890) holographic optical elements, (030.1670) coherent optical effects, (140.3298) laser beam combining, (140.5560) pumping, and (140.2050) dye lasers.

\section{Competing Interests}

The authors declare that they have no competing interests.

\section{References}

[1] R. J. Collier, B. C. Burckhardt, and H. L. Lin, Optical Holography, Academic Press, London, UK, 1971.

[2] M. Born and E. Wolf, Principles of Optics, Pergamon Press, London, UK, 1964.

[3] R. W. Ditchburn, Light, Academic Press, New York, NY, USA, 3rd edition, 1976.

[4] Z. V. Wardosanidze, A. Chanishvili, G. Petriashvili, and G. Chilaya, "Cholesteric liquid crystal holographic laser," Optics Letters, vol. 39, no. 4, pp. 1008-1010, 2014.

[5] H. Kogelnik and C. V. Shank, "Stimulated emission in a periodic structure," Applied Physics Letters, vol. 18, no. 4, pp. 152-154, 1971.

[6] J. E. Bjorkholm and C. V. Shank, "Higher-order distributed feedback oscillators," Applied Physics Letters, vol. 20, no. 8, pp. 306-308, 1972.

[7] J. E. Bjorkholm and C. V. Shank, "Distributed-feedback lasers in thin-filmoptical waveguides," Quantum Electronics, vol. 8, no. 11, pp. 833-838, 1972.

[8] V. M. Katarkevich, A. N. Rubinov, S. A. Ryzhechkin, and T. Sh. Efendiev, "Compact holographic solid-state distributedfeedback laser," Quantum Electronics A, vol. 24, no. 10, pp. 871873, 1994.

[9] N. A. Loiko and A. N. Rubinov, "Suppression of superluminescence in a dye DFB-laser with a dynamic grating," Journal of Applied Spectroscopy, vol. 67, no. 4, pp. 642-649, 2000.

[10] M. Fukuda and K. Mito, "Solid-state dye laser with photoinduced distributed feedback," Japanese Journal of Applied Physics, vol. 39, no. 10, pp. 5859-5863, 2000.

[11] Z. V. Wardosanidze, "Distributed feedback laser," Patent of Georgia no. 2780, 2002. 


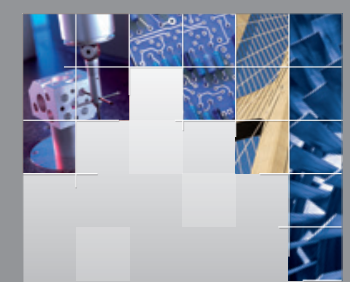

\section{Enfincering}
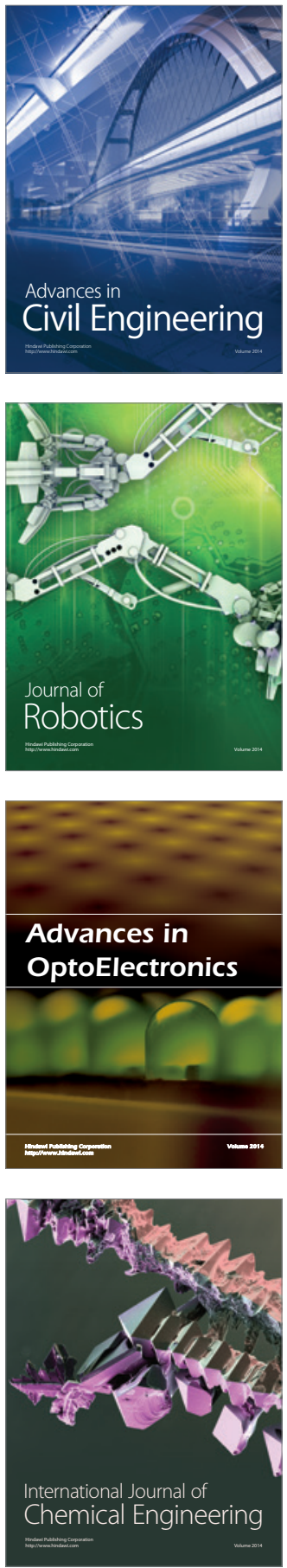

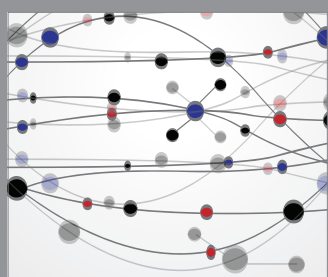

The Scientific World Journal

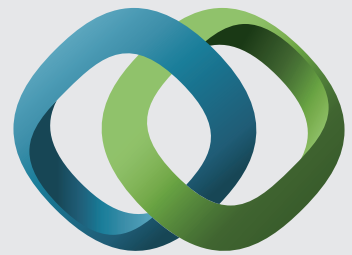

\section{Hindawi}

Submit your manuscripts at

http://www.hindawi.com
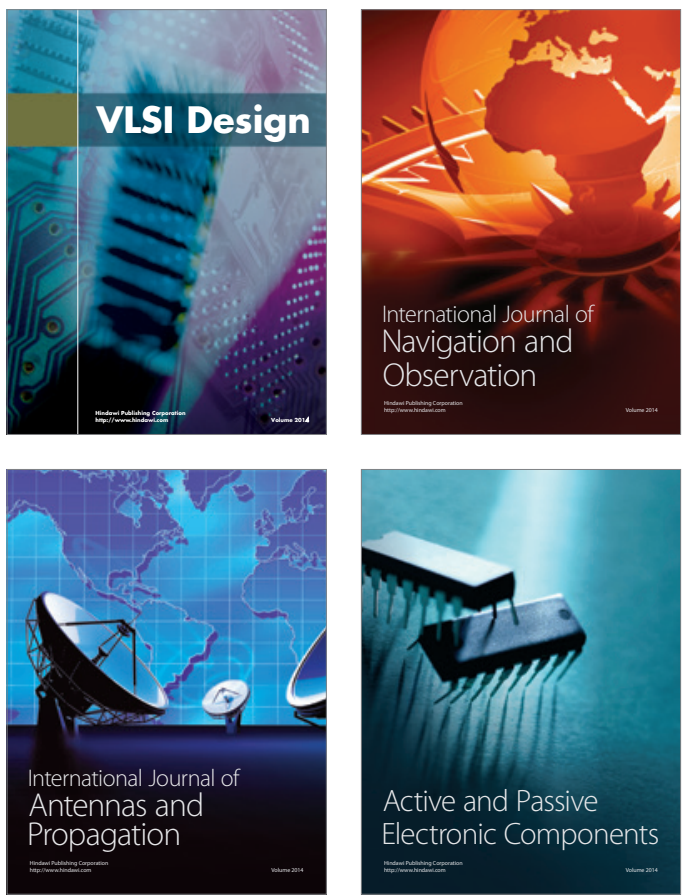
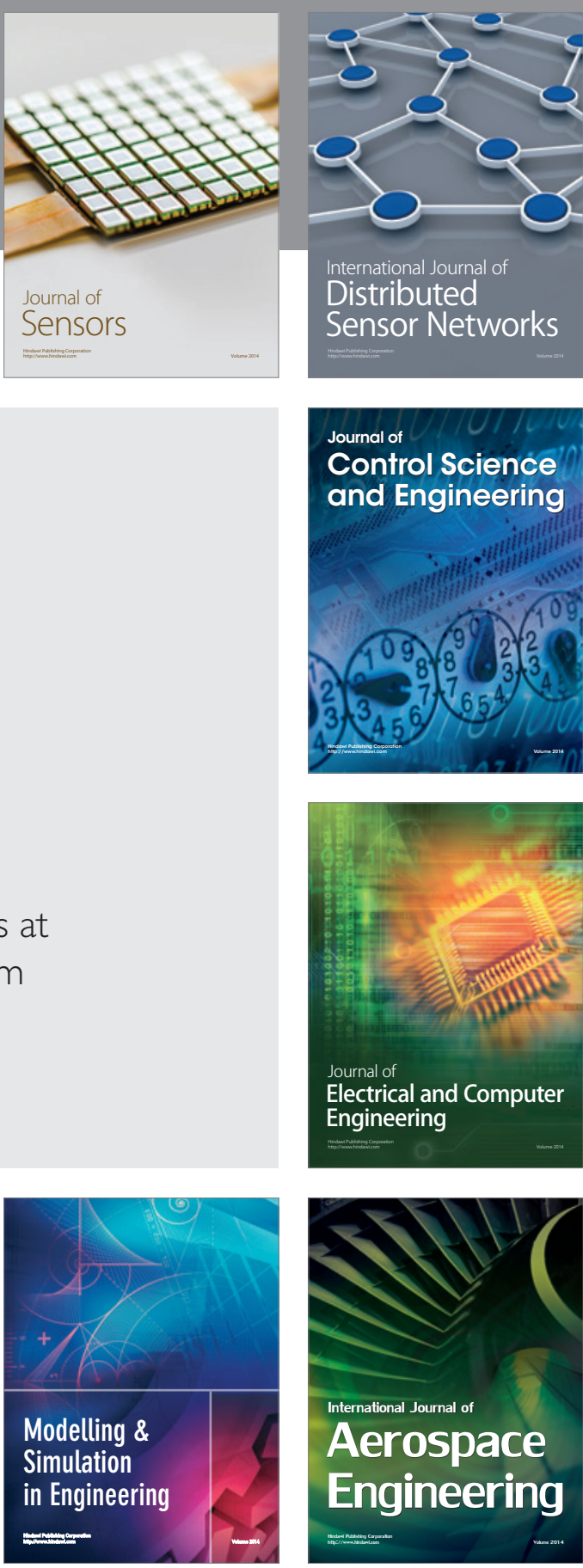

International Journal of

Distributed

Sensor Networks

Journal of

Control Science

and Engineering
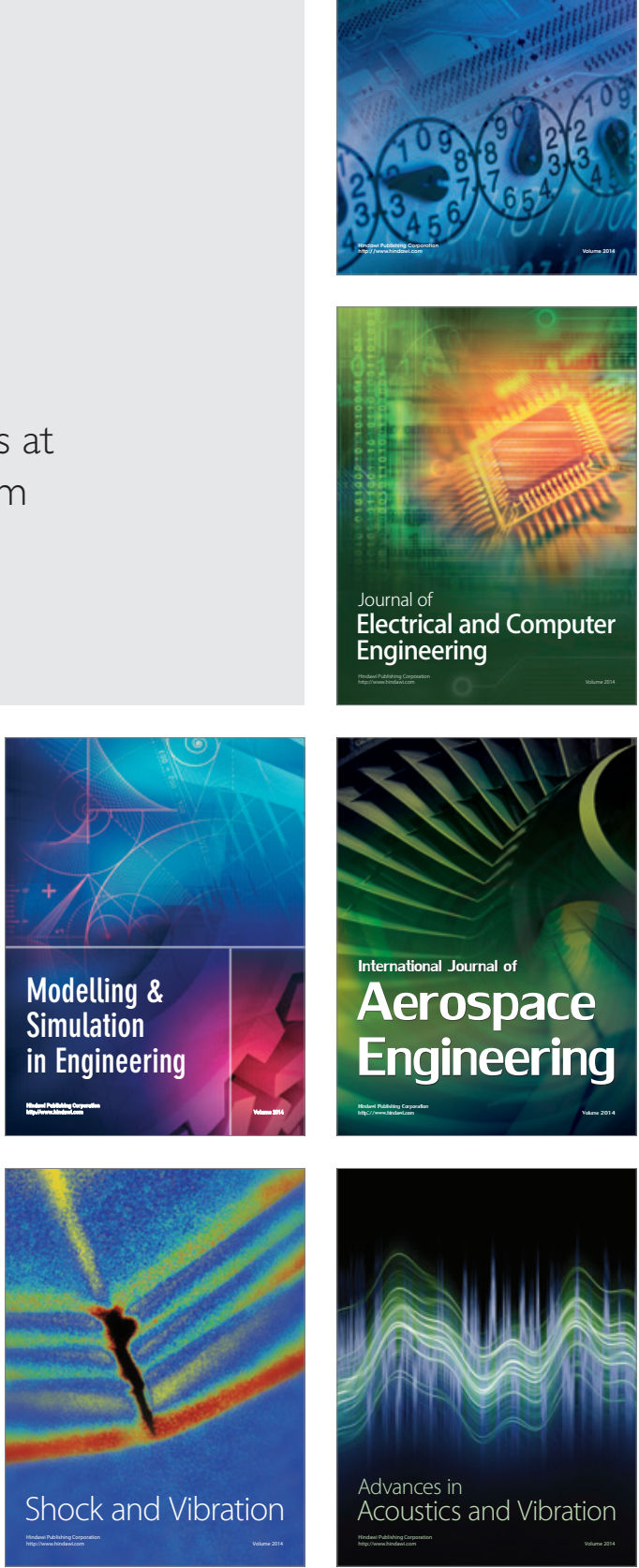